\title{
Assessment of Industrial Pollution Load in Lagos, Nigeria by Industrial Pollution Projection System (IPPS) versus Effluent Analysis
}

\author{
Adebola Oketola and Oladele Osibanjo \\ Department of Chemistry, University of Ibadan, Ibadan
}

Nigeria

\section{Introduction}

Lagos is the economic capital of Nigeria with over $70 \%$ of industries in the country located there. It is also the fastest growing city in Nigeria in terms of development and industrial infrastructure, forecast to be one of the three megacities in the world with population of over 20 million by the year 2025. The rapid growth and haphazard urbanization have led to an increase in waste generation and environmental pollution. The industrial pollution problems faced by Lagos with over 7,000 medium and large scale manufacturing facilities are directly related to the rapid industrial growth and the haphazard industrialization without environmental consideration (Oketola and Osibanjo, 2009a). Pollution abatement technologies are largely absent and the consequence is a gross pollution of natural resources and environmental media. Since effective environmental protection cannot take place in a data vacuum, Industrial Pollution Projection System (IPPS), which is a rapid environmental management tool for pollution load assessment, has been employed in this study to estimate industrial pollution loads and to ascertain the agreement between IPPS models and conventional effluent analysis.

It has been recognized that the developing countries lack the necessary information to set priorities, strategies, and action plans on environmental issues. Plant-level monitoring of air, water and toxic emissions is at best imperfect, monitoring equipment is not available and where available is obsolete; data collection and measurement methodology are questionable, and there is usually lack of trained personnel on industrial sites (Oketola and Osibanjo, 2009b; Hettige et al., 1994). In the absence of reliable pollution monitoring data, the World Bank has created a series of datasets that have given the research community the opportunity to better understand levels of pollution in developing countries, and therefore issue policy advice with more clarity (Aguayo et al., 2001). Hence, the World Bank developed the Industrial Pollution Projection System (IPPS), which is a rapid assessment tool for pollution load estimation towards the development of appropriate policy formulation for industrial pollution control in the developing countries, where insufficient data on industrial pollution proved to be an impediment to setting-up pollution control strategies and prioritization of activities (Faisal, 1991; Arpad et al, 1995).

IPPS is a modeling system, which has been developed to exploit the fact that industrial pollution is heavily affected by the scale of industrial activity, by its sectoral composition, and by the type of process technology used in production. IPPS combines data from 
industrial activities (such as production and employment) with data on pollution emissions to calculate the pollution intensity factors based on the International Standard Industrial Classification (ISIC) (Hettige et al., 1994). The IPPS has been estimated from massive USA database. This database was created by merging manufacturing census data with USEPA data on air, water, and solid waste emissions. It draws on environmental, economic, and geographic information from about 200,000 US factories. The IPPS covers about 1,500 product categories, all operating technologies, and hundreds of pollutants. It can project air, water, or solid waste emissions, and it incorporates a range of risk factors for human toxic and ecotoxic effects (Hettige et al., 1995).

There are wide ranges of industries and the pollutants introduced largely depends on the type of industry, raw material characteristics, specific process methods, efficacy of facilities, operating techniques, product grades and climatic conditions (Onianwa, 1985). The industrial sectors in Lagos based on the Manufacturer's Association of Nigeria (M.A.N) grouping are food, beverage and tobacco; textile, wearing apparel; pulp and paper products; chemical and pharmaceutical; wood and wood products; nonmetallic mineral products; basic metal; electrical and electronic; motor vehicle and miscellaneous; and domestic and industrial plastics (M.A.N., 1991).The Chemical and pharmaceutical sector is the most polluting industrial sector out of the ten major sectors based on the final ranking of IPPS pollution loads estimated with respect to employment and total value of output while basic metal, domestic and industrial plastics and textile wearing apparel sectors followed suit (Oketola and Osibanjo, 2009a). The chemical manufacturing facilities in the sector range from paint manufacturing industries, soap and detergents, pharmaceuticals, domestic insecticides and aerosol, petroleum products, toiletries and cosmetics, basic industrial chemicals while the basic metal manufacturing facilities are steel manufacturing, metal fabrication, aluminium extrusion etc.

The magnitude of environmental pollution problem is related to the types and quantity of waste generated by industries and the methods of management of the waste. As indicated earlier, there are over 7,000 industries in Lagos state with less than $10 \%$ having installed treatment facilities (Onyekwelu et al., 2003). Majority of these industries discharge their partially treated or untreated effluents into the environment and the Lagos Lagoon has gradually become a sink for pollutants from these industries. Industries utilize water for many purposes; these include processing, washing, cooling, boiler use, flushing sanitary/sewage use and general cleaning. Very large amount of water is required for these activities.

Within a given industrial sector, water use correlates with the size of the industry, and also for predicting the rate of generation of wastewater. Water supply requirements of an industry vary from one sector to another. While some industries may only require smaller volumes for cooling and cleaning (as in metal fabrication, cement bagging, etc), some others due to the nature of their processes may require very large volumes of water. Among such industries are breweries, distilleries and soft drinks manufacturing industries where water forms the bulk of the products themselves as a solution. Total consumption is about 205,000 $\mathrm{m}^{3} /$ day, with major users being Breweries, 22\%; Textile, 18\%; and Industrial chemicals, $16.6 \%$ (M.A.N., 2003). Industries utilize a vast array of input in the process of production of goods and services, and generate different forms of waste to varying degrees, which depends on the types and quantity of raw materials inputs, and the process technology employed (Ogungbuyi and Osho, 2005).

This study estimated pollution loads of some industries among the top most polluting sectors in Lagos (i.e., chemical, basic metal, plastics and textile). The selection of the 
industries was based on data availability and level of cooperation by industries studied. The industries selected are paint manufacturing, industrial gas manufacturing and lubricating oil production under the chemical and pharmaceutical sector while aluminium extrusion, steel manufacturing and glass bottle cap production industries were selected under the basic metal sector. Tyre manufacturing, foam and plastic production; and textile fabric and yarn production industries were selected under the domestic and industrial plastics and textile and wearing apparel sectors, respectively. IPPS pollution loads were estimated with respect to employment and total output, and the results of effluent pollution loads were compared statistically with IPPS pollution loads.

\section{Experimental}

\subsection{Description of the study area}

Lagos state has the largest population density of the four most industrialized states in Nigeria (Lagos, Rivers, Kano and Kaduna). It is also the state with the greatest concentration of industries, with well over seven thousand medium and large-scale industrial establishments. It is claimed that about $70-80 \%$ of the manufacturing facilities operating within the medium and large-scale industries are located there in. The major industrial estates in Lagos are: Ikeja, Agidingbi, Amuwo Odofin (industrial), Apapa, Gbagada, Iganmu, Ijora, Ilupeju, Matori, Ogba, Oregun, Oshodi/Isolo/Ilasamaja, Surulere (light industrial) and Yaba (Arikawe, 2002; Akinsanya, 2003; Ogungbuyi and Osho, 2005) as shown in Fig. 1.

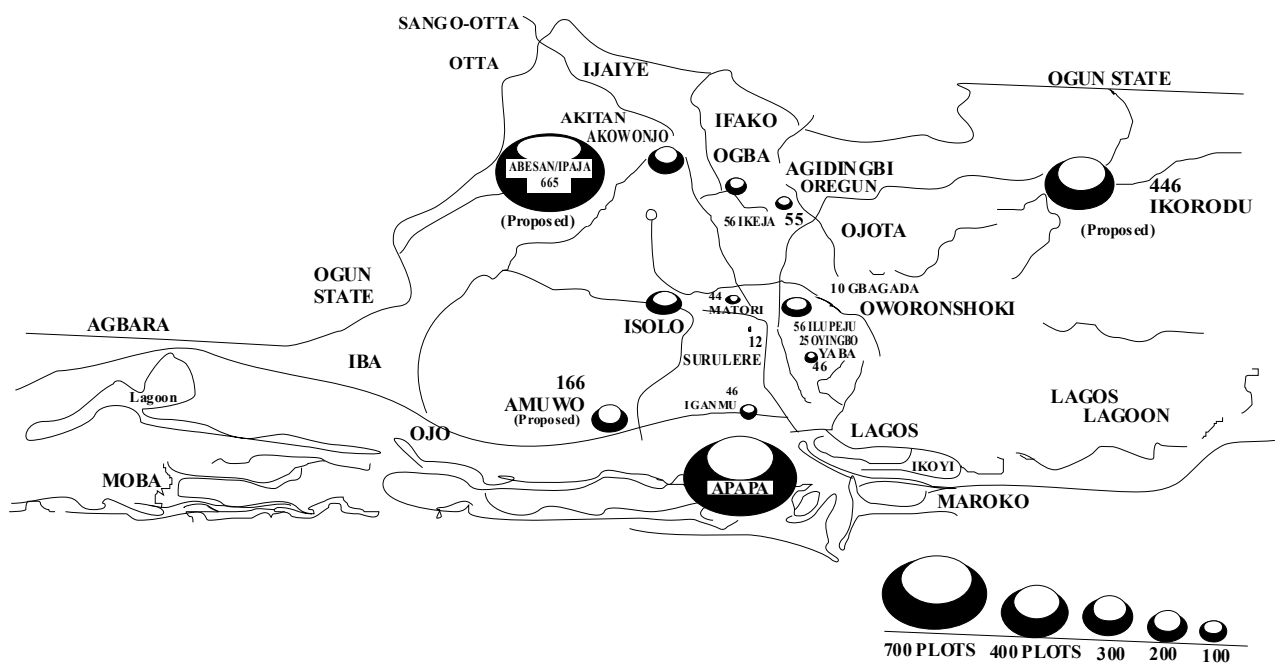

Fig. 1. Map of Industrial Estates in Lagos

\subsection{Pollution data estimation methodology}

Economic considerations and lack of cooperation from the industries limited the selection of number of industries considered in this study and the number of samples analysed. Hence, two paint manufacturing industries represented as CAP and BGR, domestic insecticides and 
aerosol production (DIA), and basic industrial gas manufacturing (IGM) were considered under the chemical and pharmaceutical sector; steel manufacturing (UST), aluminium extrusion (AET), aluminium windows and doors production (AWD) and glass bottle cap production $(\mathrm{CCP})$ were selected under the basic metal sector. Industries selected under the domestic and industrial plastics and textile and wearing apparels were tyre, foam and plastic manufacturing industries; and textile and yarn manufacturing industries, respectively.

The total number of employees and average total output in CAP, BGR, LOP, UST, CCM, AWD, AET, FMI, TTP, CLP, WSY, RLT and APT were 225 and 3, 900 ton/yr; 250 and 8,000 ton/yr; 200 and 16.1 ton/yr; 120 and 1,170 ton/yr; 1,025 and 63,200 ton/yr; 370 while total output data was not available; 36 and 222 ton/yr; 200 and 1,800 ton/yr; 710 and 6,650 ton/yr; 1,000 and 9,560 ton/yr; 200 and 960,000 ton/yr; 350 and 12,000 ton/yr; 800 and 3,600 ton/yr; and 375 and 3,750 ton/yr, respectively. Lower Bound (LB) pollution intensities by medium with respect to total value of output and employment were obtained from the literature (Hettige, et al., 1994). The pollution intensities were used to estimate the pollution loads of these manufacturing industries based on the International Standard Industrial Classification (ISIC) code as found in the literature using the formulae:

With respect to total output;

$$
\text { Pollution load }=\frac{\text { Pollution intensity factor } x \text { Unit of Output }}{2204.6}
$$

With respect to employment;

$$
\mathrm{PL}=\frac{\mathrm{PI} \times \mathrm{TEM}}{1000 \times 2204.6}
$$

Where,

$\mathrm{PL}=\quad$ Pollution load of a sector in ton/year

PI = Pollution intensity per thousand employees per year

TEM $=$ Total number of employees in that sector

$2204.6=$ Conversion factor from pounds to tonnes

\subsection{Effluent sample analysis}

Treated and untreated effluent samples were collected from the industries at the point of discharge to the environment and production line, respectively. Effluent samples were analyzed for physico-chemical parameters and heavy metals using standard methods (APHA, 1992; Miroslav and Viadimir, 1999; Taras, 1950). The parameters determined were: temperature, $\mathrm{pH}$, turbidity, conductivity, total suspended solids (TSS), total hardness, acidity, alkalinity, chloride, sulphate, nitrate, chemical oxygen demand (COD), biological oxygen demand (BOD), dissolved oxygen (DO), sodium chloride, calcium, magnesium, and heavy metals (e.g., $\mathrm{Fe}, \mathrm{Pb}, \mathrm{Zn}, \mathrm{Cd}, \mathrm{Cr}, \mathrm{Mn}, \mathrm{Ni}, \mathrm{Cu}$, and $\mathrm{Co}$ ).

\subsection{Statistical analysis}

The data were validated statistically using $\mathrm{t}$ - test at $95 \%$ confidence interval (2- tailed) and analysis of variance (ANOVA) to ascertain if there is any significant difference between IPPS pollution loads with respect to employment and total output; and pollution loads from conventional effluent analysis at $\mathrm{p}>0.05$. 


\begin{tabular}{|c|c|c|c|c|c|c|c|}
\hline $\begin{array}{c}\text { Industrial } \\
\text { Sector }\end{array}$ & $\begin{array}{l}\text { Four } \\
\text { ISIC } \\
\text { Code } \\
\end{array}$ & $\begin{array}{l}\text { Product } \\
\text { Produced }\end{array}$ & $\begin{array}{l}\text { Major Raw } \\
\text { Materials }\end{array}$ & $\begin{array}{c}\text { Types of } \\
\text { Waste } \\
\text { Generated } \\
\end{array}$ & $\begin{array}{l}\text { Mode of } \\
\text { Disposal }\end{array}$ & \begin{tabular}{|c|} 
Effluent \\
Treatment Plant \\
(ETP)/Constrain \\
\end{tabular} & General Remarks \\
\hline \multirow{4}{*}{$\mathrm{CPH}$} & $\begin{array}{l}3521 \\
(\mathrm{CAP})\end{array}$ & Paints & $\begin{array}{l}\text { Pigment, resin, } \\
\text { solvent and } \\
\text { additives }\end{array}$ & $\begin{array}{l}\text { Effluent } \\
\text { Waste } \\
\text { solvent }\end{array}$ & $\begin{array}{l}\text { Discharge in } \\
\text { drain } \\
\text { By contractor } \\
\text { off-site }\end{array}$ & Operational & $\begin{array}{l}\text { Discharge treated } \\
\text { effluent into the } \\
\text { environment }\end{array}$ \\
\hline & $\begin{array}{c}3521 \\
(\mathrm{BGR})\end{array}$ & \begin{tabular}{|c|} 
Paints, \\
wood \\
preservative \\
s, allied \\
products
\end{tabular} & \begin{tabular}{|} 
Dyes, pigment, \\
solvent, \\
extender
\end{tabular} & $\begin{array}{l}\text { Effluent } \\
\text { Sludge }\end{array}$ & $\begin{array}{l}\text { Discharge in } \\
\text { drain } \\
\text { By contractor } \\
\text { off-site }\end{array}$ & Operational & $\begin{array}{l}\text { Discharge treated } \\
\text { effluent into the } \\
\text { environment }\end{array}$ \\
\hline & $\begin{array}{c}3511 \\
(\mathrm{IGM})\end{array}$ & $\begin{array}{c}\text { Industrial } \\
\text { gases e.g. } \\
\mathrm{O}_{2}, \mathrm{CO}_{2} \\
\text { acetylene }\end{array}$ & \begin{tabular}{|} 
Caustic soda, \\
soda ash, \\
calcium carbide, \\
ammonium \\
nitrate.
\end{tabular} & $\begin{array}{l}\text { Effluent, } \\
\text { Sludge }\end{array}$ & \begin{tabular}{|c|} 
Discharge in \\
drain, \\
Sludge is \\
disposed by \\
contractor off- \\
site \\
\end{tabular} & $\begin{array}{l}\text { Not available, } \\
\text { installing ETP }\end{array}$ & $\begin{array}{l}\text { Discharge effluent } \\
\text { to the environment }\end{array}$ \\
\hline & $\begin{array}{l}3540 \\
(\mathrm{LOP})\end{array}$ & $\begin{array}{c}\text { Lubricants, } \\
\text { aerosol } \\
\text { insecticide } \\
\text { etc }\end{array}$ & $\begin{array}{l}\text { Petroleum } \\
\text { products }\end{array}$ & $\begin{array}{l}\text { Effluent } \\
\text { Solid waste } \\
\text { Sludge }\end{array}$ & $\begin{array}{c}\text { Used oil } \\
\text { generated is } \\
\text { discharged to } \\
\text { cement kiln and } \\
\text { solid/sludge by } \\
\text { contractor off } \\
\text { site } \\
\end{array}$ & Operational & $\begin{array}{c}\text { Treat effluent before } \\
\text { discharge }\end{array}$ \\
\hline \multirow{3}{*}{ DIP } & $\begin{array}{l}3551 \\
(\mathrm{TTP})\end{array}$ & $\begin{array}{c}\text { Tyres for } \\
\text { cars, trucks } \\
\text { and light } \\
\text { trucks }\end{array}$ & \begin{tabular}{|c|} 
Natural and \\
synthetic \\
rubber, $\mathrm{ZnO}$, \\
cobalt stearate, \\
carbon black, \\
mineral oil \\
\end{tabular} & $\begin{array}{c}\text { Effluent } \\
\text { Solid waste }\end{array}$ & $\begin{array}{l}\text { Discharge in } \\
\text { drain, } \\
\text { By contractor } \\
\text { off-site }\end{array}$ & Not available & $\begin{array}{l}\text { Uses effluent as } \\
\text { cooling water }\end{array}$ \\
\hline & $\begin{array}{l}3513 \\
\text { (FMI) }\end{array}$ & \begin{tabular}{|c|}
$\begin{array}{c}\text { Flexible and } \\
\text { rigid foams, } \\
\text { adhesives }\end{array}$ \\
\end{tabular} & \begin{tabular}{|c|} 
Polyol, toluene- \\
di-isocyanate \\
(IDI), silicone \\
oil, methylene \\
chloride \\
\end{tabular} & Solid waste & Recycled & Not available & $\begin{array}{c}\text { Emitting volatile } \\
\text { organic compounds } \\
\text { into the atmosphere }\end{array}$ \\
\hline & $\begin{array}{c}3560(\mathrm{CL} \\
\mathrm{P})\end{array}$ & Plastics & $\begin{array}{l}\text { Pigments and } \\
\text { mastic batches }\end{array}$ & Solid waste & $\begin{array}{c}\text { Waste oil } \\
\text { discharged by } \\
\text { contractor off- } \\
\text { site }\end{array}$ & Not Applicable & $\begin{array}{l}\text { Do not generate } \\
\text { effluent at the } \\
\text { production line }\end{array}$ \\
\hline \multirow{3}{*}{ TWA } & $\begin{array}{l}3211 \\
(\mathrm{RLT})\end{array}$ & $\begin{array}{c}\text { Grey fabrics } \\
\text { e.g. suiting, } \\
\text { ankara }\end{array}$ & $\begin{array}{c}\text { Yarn, chemicals } \\
\text { and dyes }\end{array}$ & $\begin{array}{c}\text { Effluent } \\
\text { Solid waste }\end{array}$ & $\begin{array}{l}\text { In drain after } \\
\text { treatment } \\
\text { By contractor } \\
\text { off-site }\end{array}$ & Operational & $\begin{array}{l}\text { Discharge treated } \\
\text { effluent into the } \\
\text { environment }\end{array}$ \\
\hline & $\begin{array}{c}3211 \\
\text { (WSY) }\end{array}$ & Textiles & $\begin{array}{c}\text { Dyes, pigment, } \\
\text { caustic soda, } \\
\text { acetic acid }\end{array}$ & $\begin{array}{c}\text { Effluent } \\
\text { Solid waste }\end{array}$ & $\begin{array}{c}\text { Discharge in } \\
\text { drain, by } \\
\text { contractor off- } \\
\text { site } \\
\end{array}$ & Operational & $\begin{array}{l}\text { Discharge treated } \\
\text { effluent into the } \\
\text { environment }\end{array}$ \\
\hline & $\begin{array}{c}3219 \\
(\mathrm{APT})\end{array}$ & Yarn & Cotton & Solid waste & $\begin{array}{l}\text { By contractor } \\
\text { off-site }\end{array}$ & Not applicable & $\begin{array}{l}\text { Do not generate } \\
\text { effluent. }\end{array}$ \\
\hline \multirow[t]{2}{*}{ BML } & $\begin{array}{c}3720 \\
(\mathrm{AET})\end{array}$ & $\begin{array}{c}\text { Aluminium } \\
\text { profiles }\end{array}$ & $\begin{array}{c}\text { Aluminium } \\
\text { billets, } \mathrm{H}_{2} \mathrm{SO}_{4}, \\
\mathrm{NaOH}_{4} \mathrm{Tin}(\mathrm{II}) \\
\text { Sulphate, } \\
\text { Chromic acid }\end{array}$ & $\begin{array}{l}\text { Effluent, } \\
\text { solid and } \\
\text { sludge }\end{array}$ & $\begin{array}{c}\text { Effluent } \\
\text { discharged in } \\
\text { drain after } \\
\text { treatment and } \\
\text { sludge by } \\
\text { contractor off- } \\
\text { site. } \\
\end{array}$ & ETP operational & $\begin{array}{c}\text { Do not discharge } \\
\text { effluent that } \\
\text { contains hazardous } \\
\text { substances into the } \\
\text { environment. }\end{array}$ \\
\hline & $\begin{array}{c}3720 \\
(\mathrm{AWD})\end{array}$ & \begin{tabular}{|c|} 
Aluminium \\
windows
\end{tabular} & $\begin{array}{l}\text { Aluminium } \\
\text { profile from }\end{array}$ & Solid waste & Recycle waste & Not applicable & $\begin{array}{l}\text { Do not generate } \\
\text { effluent at all. }\end{array}$ \\
\hline
\end{tabular}




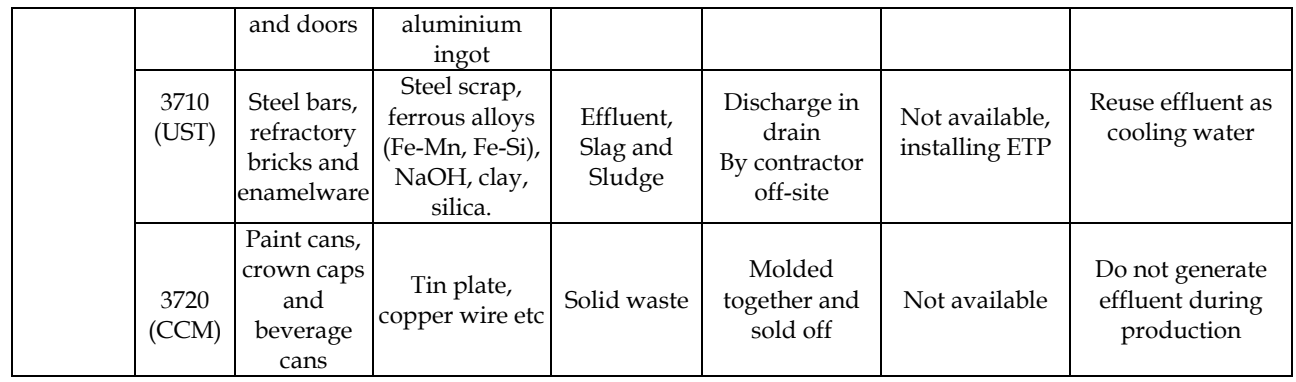

Table 1. Major raw materials and types of waste generated by the selected industries in Lagos

\section{Results and discussion}

Emission to air was determined based on emission of total suspended particulate (TSP), fine particulate (FP, PM10), sulphur dioxide $\left(\mathrm{SO}_{2}\right)$, nitrogen dioxide $\left(\mathrm{NO}_{2}\right)$, carbon monoxide (CO), and volatile organic compounds (VOCs). Emission to water was estimated in terms of biological oxygen demand (BOD) and total suspended solid (TSS) while emission of toxic pollutants was estimated in terms of toxic chemicals and metals released into air, water and land, whose pollution intensities were available in the literature (Hettige, et al., 1994). The major raw materials and the type of waste generated by the selected industries are presented in Table 1 while the total number of employees and total value of output as well as the pollution loads are shown in Tables 2 and 3, respectively. UST have the highest number of employees and second highest total value of output while AWD have the lowest number of employees and LOP the lowest value of output.

\subsection{IPPS pollution load assessment}

\subsubsection{Air pollution load}

Air pollution loads for all the selected industries are shown in Tables 2 and 3, respectively for pollution load estimated with respect to employment and total value of output. UST with 1025 employees and 63, 200 ton/yr of total output have the highest emission of all pollutants into environmental media (i.e., air, water, and land). The air pollution load with respect to employment and total value of output are 4,810 tons/yr and 1,860,000 tons/yr, respectively. This was followed by FMI,CCM, LOP, AET, TTP, IGM, RLT, APT, AWD, WSY, $\mathrm{BGR}, \mathrm{CAP}$, and CLP, respectively in decreasing order.

In most cases, the higher the number of employees and total output, the higher the air pollution loads. Basic metal, and domestic and industrial plastic (DIP) sectors are the most polluting sector in terms of air pollutant emission. UST ranked first while FMI and CCM ranked second and third, respectively. Total air pollution loads with respect to employment are 2,660 tons/yr and 2050 tons/yr in FMI and CCM, respectively. With respect to total output, air pollution loads are 94,500 ton/yr in FMI. Output data from CCM was not available thus; air pollution load with respect to total output cannot be estimated. Emission of $\mathrm{CO}$ and $\mathrm{NO}_{2}$ was the highest in UST and FMI when pollution load was estimated with respect to the two variables (i.e., employment and total output) while $\mathrm{SO}_{2}$ emission was the highest in CCM when pollution load was estimated with respect to employment. The trend in air pollution load by pollutant types in these industries are

$$
\text { UST: } \mathrm{CO}>\mathrm{SO}_{2}>\mathrm{NO}_{2}>\mathrm{FP}>\mathrm{TSP}>\mathrm{VOC}
$$


FMI: $\mathrm{NO}_{2}>\mathrm{VOC}>\mathrm{SO}_{2}>\mathrm{CO}>\mathrm{TSP}>\mathrm{FP}$

CCM: $\mathrm{SO}_{2}>\mathrm{CO}>\mathrm{TSP}>\mathrm{VOC}>\mathrm{NO}_{2}>\mathrm{FP}$

Pollution loads estimated with respect to employment and total output revealed that the most emitted air pollutant from UST was CO. This could be attributed to the fact that in steel making, oxygen reacts with several components in the bath, including $\mathrm{Al}, \mathrm{Si}, \mathrm{Mn}, \mathrm{P}, \mathrm{C}$, and $\mathrm{Fe}$, to produce metallic oxides which end up in the slag. It also generates carbon monoxide boil, a phenomenon common to all steel making processes and very important for mixing of the slag. Mixing enhances chemical reaction, purges hydrogen and nitrogen, and improves heat transfer. The $\mathrm{CO}$ supplies a less expensive form of energy to the bath, and performs several important refining reactions (Jeremy, 2003; and Bruce and Joseph, 2003). It is also important for foaming and help to bury the arc.

\begin{tabular}{|c|c|c|c|c|c|c|c|c|}
\hline $\begin{array}{l}\text { INDUSTRIAL } \\
\text { SECTOR/ } \\
\text { SECTOR CODE }\end{array}$ & \multicolumn{4}{|c|}{$\begin{array}{l}\text { CHEMICAL \& PHARMACEUTICALS } \\
\text { (CPH) }\end{array}$} & \multicolumn{4}{|c|}{ BASIC METALS (BML) } \\
\hline ISIC CODE & $\begin{array}{l}3521 \\
\text { (CAP) }\end{array}$ & $\begin{array}{l}3521 \\
\text { (BGR) }\end{array}$ & $\begin{array}{l}3540 \\
(\mathrm{LOP})\end{array}$ & $\begin{array}{l}3511 \\
(\mathrm{IGM})\end{array}$ & $\begin{array}{l}3710 \\
\text { (UST) }\end{array}$ & $\begin{array}{l}3720 \\
(\mathrm{CCM})\end{array}$ & $\begin{array}{l}3720 \\
(\mathrm{AWD})\end{array}$ & $\begin{array}{l}3720 \\
(\mathrm{AET})\end{array}$ \\
\hline $\begin{array}{l}\text { EFFLUENT VOL. } \\
\text { (L/day) }\end{array}$ & 1,500 & 2,000 & NA & NA & 1MILLON & $\mathrm{NA}^{*}$ & NA & 10 \\
\hline $\begin{array}{l}\text { EFFLUENT } \\
\text { TREATMENT PLANT } \\
(\text { ETP })\end{array}$ & \begin{tabular}{|l|} 
Operational \\
\end{tabular} & Operational & Operational & NA & NA & $\mathrm{NA}^{*}$ & NA & Operational \\
\hline NO OF EMPLOYEE & $225(\mathrm{M})$ & $250(\mathrm{M})$ & $200(\mathrm{M})$ & $120(\mathrm{M})$ & $1025(\mathrm{~L})$ & $370(\mathrm{M})$ & $36(\mathrm{M})$ & $200(\mathrm{M})$ \\
\hline \multicolumn{9}{|l|}{ AIR POLLUTANTS } \\
\hline $\mathrm{SO}_{2}$ & 5.88 & 6.53 & 565 & 200 & 1320 & 1,260 & 122 & 680 \\
\hline $\mathrm{NO}_{2}$ & 5.19 & 5.77 & 352 & 148 & 575 & 41.0 & 3.99 & 22.2 \\
\hline $\mathrm{CO}$ & 0.73 & 0.81 & 266 & 115 & 2060 & 586 & 57.0 & 317 \\
\hline VOC & 43.5 & 48.4 & 88.3 & 116 & 177 & 45.8 & 4.46 & 24.8 \\
\hline FP & 1.78 & 1.98 & 17.4 & 6.77 & 366 & 11.6 & 1.13 & 6.25 \\
\hline TSP & 3.49 & 3.88 & 217 & 32.1 & 307 & 106 & 10.3 & 57.2 \\
\hline TOTAL & 60.6 & 67.3 & 1,510 & 617 & 4810 & 2,050 & 199 & 1,110 \\
\hline \multicolumn{9}{|l|}{$\begin{array}{l}\text { WATER } \\
\text { POLLUTANTS }\end{array}$} \\
\hline BOD & 0.01 & 0.07 & 0.59 & 68.3 & 0.89 & 96.5 & 9.39 & 52.2 \\
\hline TSS & 0.03 & 0.03 & 0.73 & 105.6 & 14,400 & 1,400 & 136 & 754 \\
\hline TOTAL & 0.04 & 0.10 & 1.32 & 174 & 14,400 & 1,490 & 145 & 806 \\
\hline \multicolumn{9}{|l|}{ TOXIC CHEMICALS } \\
\hline TO AIR & 38.8 & 43.1 & 10.8 & 101 & 73.0 & 97.3 & 9.47 & 52.6 \\
\hline TO LAND & 93.1 & 103 & 3.17 & 353 & 418 & 258 & 25.1 & 140 \\
\hline TO WATER & 0.10 & 0.11 & 0.32 & 51.3 & 25.9 & 3.78 & 0.38 & 2.04 \\
\hline TOTAL & 132 & 147 & 14.7 & 505 & 517 & 359 & 35.0 & 194 \\
\hline \multicolumn{9}{|l|}{ TOXIC METALS } \\
\hline TO AIR & 0.33 & 0.37 & 0.02 & 0.50 & 12.5 & 6.73 & 0.66 & 3.64 \\
\hline TO LAND & 2.54 & 2.82 & 0.30 & 15.9 & 276 & 223 & 21.7 & 121 \\
\hline TO WATER & 0.002 & 0.002 & 0.01 & 0.47 & 1.89 & 0.13 & 0.01 & 0.07 \\
\hline TOTAL & 2.89 & 3.18 & 0.33 & 16.9 & 291 & 230 & 22.4 & 124 \\
\hline
\end{tabular}

NOTE: $\mathrm{L}=$ large scale, $\mathrm{M}=$ medium scale, $\mathrm{S}=$ small scale, $\mathrm{NA}=$ not available, $\mathrm{NA}^{*}=$ not applicable

Table 2. Pollution loads (ton/yr) with respect to employment 


\begin{tabular}{|c|c|c|c|c|c|c|}
\hline \multirow{2}{*}{$\begin{array}{l}\text { INDUSTRIAL } \\
\text { SECTOR/SECTOR CODE } \\
\text { ISIC CODE }\end{array}$} & \multicolumn{3}{|c|}{$\begin{array}{l}\text { DOMESTIC AND INDUSTRIAL } \\
\text { PLASTICS } \\
\text { (DIP) }\end{array}$} & \multicolumn{3}{|c|}{ TEXTILE, WEARING APPAREL (TWA) } \\
\hline & 3560 (CLP) & 3513 (FMI) & 3551 (TTP) & & \begin{tabular}{|l|}
3219 \\
(APT)
\end{tabular} & 3211 (RLT) \\
\hline EFFLUENT VOL. (L/day) & $\mathrm{NA}^{*}$ & $\mathrm{NA}^{*}$ & 484,000 & 160 & $\mathrm{NA}^{*}$ & 720 \\
\hline $\begin{array}{l}\text { EFFLUENT TREATMENT } \\
\text { PLANT (ETP) }\end{array}$ & NA & NA & NA & $\begin{array}{l}\text { Operatio } \\
\text { nal }\end{array}$ & NA & $\begin{array}{l}\text { Operationa } \\
1\end{array}$ \\
\hline NO OF EMPLOYEE & $200(\mathrm{M})$ & $710(\mathrm{~L})$ & $1,000(\mathrm{~L})$ & $350(\mathrm{M})$ & $375(\mathrm{M})$ & $800(\mathrm{~L})$ \\
\hline \multicolumn{7}{|l|}{ AIR POLLUTANTS } \\
\hline $\mathrm{SO} 2$ & 0.54 & 441 & 275 & 36.0 & 21.0 & 82.3 \\
\hline NO2 & 0.12 & 1,150 & 95.1 & 49.7 & 8.67 & 114 \\
\hline $\mathrm{CO}$ & 0.04 & 169 & 11.7 & 6.67 & 1.58 & 15.3 \\
\hline VOC & 6.48 & 838 & 278 & 13.6 & 166 & 31.2 \\
\hline FP & 0.11 & 0.36 & 3.93 & 0.96 & 0.00 & 2.20 \\
\hline TSP & 0.16 & 67.3 & 30.4 & 6.45 & 12.5 & 14.7 \\
\hline TOTAL & 7.45 & 2,660 & 695 & 113 & 210 & 259 \\
\hline \multicolumn{7}{|l|}{ WATER POLLUTANTS } \\
\hline BOD & 4.97 & 1.89 & 0.002 & 1.46 & 0.00 & 3.34 \\
\hline TSS & 0.11 & 58.2 & 0.68 & 2.27 & 0.09 & 5.18 \\
\hline TOTAL & 5.08 & 60.0 & 0.68 & 3.73 & 0.09 & 8.52 \\
\hline \multicolumn{7}{|l|}{ TOXIC CHEMICALS } \\
\hline TO AIR & 18.2 & 484 & 9.98 & 5.22 & 147 & 11.9 \\
\hline TO LAND & 5.38 & 401 & 17.2 & 4.85 & 33.2 & 11.1 \\
\hline TO WATER & 0.04 & 35.4 & 0.21 & 2.66 & 0.01 & 6.08 \\
\hline TOTAL & 23.6 & 920 & 27.4 & 12.7 & 180 & 29.1 \\
\hline \multicolumn{7}{|l|}{ TOXIC METALS } \\
\hline TO AIR & 0.004 & 0.13 & 0.39 & 0.04 & 0.03 & 0.10 \\
\hline TO LAND & 0.16 & 20.9 & 15.1 & 0.09 & 0.01 & 0.20 \\
\hline TO WATER & 0.01 & 0.44 & 0.02 & 0.003 & - & 0.01 \\
\hline TOTAL & 0.18 & 21.5 & 15.5 & 0.13 & 0.04 & 0.31 \\
\hline
\end{tabular}

NOTE: $\mathrm{L}=$ large scale, $\mathrm{M}=$ medium scale, $\mathrm{S}=$ small scale, $\mathrm{NA}=$ not available, $\mathrm{NA}^{*}=$ not applicable Table 2. Contd. Pollution loads (ton/yr) with respect to employment 


\begin{tabular}{|c|c|c|c|c|c|c|c|c|}
\hline \multirow{2}{*}{\begin{tabular}{|l} 
INDUSTRIAL \\
SECTOR/ \\
SECTOR CODE
\end{tabular}} & \multicolumn{4}{|c|}{$\begin{array}{l}\text { CHEMICAL \& PHARMACEUTICALS } \\
\text { (CPH) }\end{array}$} & \multicolumn{4}{|c|}{ BASIC METALS (BML) } \\
\hline & 3521 (CAP) & 3521 (BGR) & 3540 (LOP) & 3511 (IGM) & 3710 (UST) & $3720(\mathrm{CCM})$ & 3720 (AWD) & 3720 (AET) \\
\hline $\begin{array}{l}\text { EFFLUENT VOL. } \\
\text { (L/day) }\end{array}$ & 1,500 & 2,000 & NA & NA & $1 \mathrm{MILLION}$ & $\mathrm{NA}^{*}$ & NA & 10 \\
\hline $\begin{array}{l}\text { EFFLUENT } \\
\text { TREATMENT } \\
\text { PLANT (ETP) }\end{array}$ & Operational & Operational & Operational & NA & NA & $\mathrm{NA}^{*}$ & NA & Operational \\
\hline $\begin{array}{l}\text { TOTAL VALUE OF } \\
\text { OUTPUT (ton/yr) }\end{array}$ & 3,900 & 8,000 & 16.1 & 1,170 & 63,200 & NA & 222 & 1,800 \\
\hline \multicolumn{9}{|l|}{ AIR POLLUTANTS } \\
\hline $\mathrm{SO}_{2}$ & 435 & 893 & 152 & 6,180 & 512,000 & NA & 3,890 & 31,600 \\
\hline $\mathrm{NO}_{2}$ & 384 & 787 & 94.7 & 4,590 & 222,000 & NA & 127 & 1,030 \\
\hline $\mathrm{CO}$ & 54.8 & 112 & 71.7 & 3,550 & 798,000 & NA & 1,800 & 14,700 \\
\hline VOC & 3,220 & 6,600 & 23.8 & 3,590 & 68,600 & NA & 141 & 1,150 \\
\hline FP & 131 & 269 & 4.68 & 210 & 142,000 & NA & 35.7 & 290 \\
\hline TSP & 258 & 530 & 58.4 & 994 & 119,000 & NA & 326 & 2,650 \\
\hline TOTAL & 4,480 & 9,190 & 405 & 19,100 & $1,860,000$ & NA & 6,320 & 51,300 \\
\hline \multicolumn{9}{|l|}{$\begin{array}{l}\text { WATER } \\
\text { POLLUTANTS }\end{array}$} \\
\hline BOD & 0.46 & 0.94 & 0.16 & 2,120 & 379 & NA & 298 & 2,410 \\
\hline TSS & 1.91 & 0.26 & 0.20 & 3,270 & $5,580,000$ & NA & 4,300 & 35,000 \\
\hline TOTAL & 2.37 & 1.20 & 0.36 & 5,390 & $5,580,000$ & NA & 4,600 & 37,400 \\
\hline TOXIC CHEMICALS & & & & & & NA & & \\
\hline TO AIR & 2,870 & 5,880 & 2.90 & 3,140 & 28,000 & NA & 300 & 2,440 \\
\hline TO LAND & 6,880 & 14,100 & 0.85 & 10,900 & 162,000 & NA & 796 & 6,470 \\
\hline TO WATER & 7.47 & 15.3 & 0.09 & 1,590 & 10,000 & NA & 11.7 & 94.8 \\
\hline TOTAL & 9,760 & 20,000 & 3.84 & 15,600 & 200,000 & NA & 1,110 & 9,000 \\
\hline \multicolumn{9}{|l|}{ TOXIC METALS } \\
\hline TO AIR & 24.3 & 49.9 & 0.01 & 15.6 & 4,850 & NA & 20.8 & 169 \\
\hline TO LAND & 187 & 385 & 0.17 & 493 & 107,000 & NA & 689 & 5,590 \\
\hline TO WATER & 0.15 & 0.32 & 0.002 & 14.5 & 732 & NA & 0.41 & 3.36 \\
\hline TOTAL & 212 & 435 & 0.18 & 523 & 112,000 & NA & 710 & 5,760 \\
\hline
\end{tabular}

NOTE: $\mathrm{NA}=$ not available, $\mathrm{NA}^{*}=$ not applicable

Table 3. Pollution loads (ton/yr) with respect to total value of output 


\begin{tabular}{|c|c|c|c|c|c|c|}
\hline \multirow{2}{*}{$\begin{array}{l}\text { INDUSTRIAL SECTOR/ } \\
\text { SECTOR CODE } \\
\text { ISIC CODE }\end{array}$} & \multicolumn{3}{|c|}{$\begin{array}{l}\text { DOMESTIC AND INDUSTRIAL } \\
\text { PLASTICS (DIP) }\end{array}$} & \multicolumn{3}{|c|}{ TEXTILE, WEARING APPAREL (TWA) } \\
\hline & 3560 (CLP) & 3513 (FMI) & \begin{tabular}{|l|}
3551 \\
(TTP)
\end{tabular} & 3211 (WSY) & 3211 (RLT) & 3219 (APT) \\
\hline EFFLUENT VOL. (L/day) & $\mathrm{NA}^{*}$ & $\mathrm{NA}^{*}$ & 484,000 & 160 & 720 & $\mathrm{NA}^{*}$ \\
\hline $\begin{array}{ll}\text { EFFLUENT } & \text { TREATMENT } \\
\text { PLANT (ETP) } & \end{array}$ & NA & NA & NA & Operational & Operational & NA \\
\hline $\begin{array}{l}\text { TOTAL VALUE OF OUTPUT } \\
\text { (ton/yr) }\end{array}$ & 960,000 & 6,650 & 9,560 & 12,000 & 3,600 & 3,750 \\
\hline \multicolumn{7}{|l|}{ AIR POLLUTANTS } \\
\hline $\mathrm{SO}_{2}$ & 24,400 & 15,600 & 16,500 & 13,200 & 3,950 & 1,270 \\
\hline $\mathrm{NO}_{2}$ & 5,230 & 40,600 & 5,690 & 18,300 & 5,460 & 526 \\
\hline $\mathrm{CO}$ & 0.001 & 6,010 & 698 & 2,450 & 731 & 95.3 \\
\hline VOC & 294,00 & 30,000 & 16,700 & 5,010 & 1,500 & 10,100 \\
\hline FP & 5,230 & 12.1 & 234 & 355 & 106 & 0.00 \\
\hline TSP & 7,400 & 2,390 & 1,820 & 2,360 & 707 & 757 \\
\hline TOTAL & 337,000 & 94,400 & 41,600 & 41,700 & 12,400 & 12,800 \\
\hline \multicolumn{7}{|l|}{ WATER POLLUTANTS } \\
\hline BOD & 226,000 & 638 & 0.09 & 536 & 160 & 0.00 \\
\hline TSS & 4,880 & 2,060 & 40.9 & 833 & 249 & 5.44 \\
\hline TOTAL & 231,000 & 2,700 & 41.0 & 1,370 & 409 & 5.44 \\
\hline \multicolumn{7}{|l|}{ TOXIC CHEMICALS } \\
\hline TO AIR & 826,000 & 17.2 & 598 & 1,920 & 573 & 8,940 \\
\hline TO LAND & 245,000 & 14.2 & 1,030 & 1,780 & 532 & 2,010 \\
\hline TO WATER & 2,020 & 1.25 & 12.4 & 977 & 292 & 0.08 \\
\hline TOTAL & $1,070,000$ & 32.6 & 1,640 & 4,670 & 1,400 & 10,900 \\
\hline \multicolumn{7}{|l|}{ TOXIC METALS } \\
\hline TO AIR & 192 & 4.76 & 23.2 & 15.8 & 4.72 & 1.83 \\
\hline TO LAND & 7,400 & 741.1 & 903 & 320 & 95.5 & 37.7 \\
\hline TO WATER & 416 & 15.5 & 1.16 & 1.07 & 0.32 & 0.35 \\
\hline TOTAL & 8,010 & 761 & 928 & 336 & 100.6 & 39.9 \\
\hline
\end{tabular}

NOTE: $\mathrm{NA}=$ not available, $\mathrm{NA}^{*}=$ not applicable

Table 3. Contd. Pollution loads (ton/yr) with respect to total value of output 


\subsubsection{Water pollution load}

Of all the industries, UST ranked first in terms of total water pollution load while CCM and AET ranked second and third, respectively. This was due to the fact that emission of TSS from the two industries was more than BOD. Estimated TSS pollution load from these industries are 14,400 and 1,400 ton/yr, respectively while BOD pollution load are 0.89 and 96.5 ton/yr, respectively. The steel industry with the highest number of employees generated the highest water pollution load. Thus, the higher the number of employees, the higher the water pollution loads. Pollution load estimated with respect to total output showed that 5.6 million ton/yr of TSS was generated by UST. Water pollution load estimated with respect to employment and total output revealed that emission of TSS was more than BOD in all the manufacturing facilities under the basic metal sector with UST having the highest water pollution load with respect to the two variables (i.e., employment and total output). This is shown in Tables 2 and 3, respectively. APT and CAP have the lowest water pollution load thus, their contribution to water pollution is insignificant.

\subsubsection{Toxic pollution load}

Toxic chemical and metal pollution load with respect to employment and total output are presented in Tables 2 and 3, respectively. Total chemical pollution load with respect to employment and total output is more than total metal pollution load in all the facilities. This may be attributed to the nature of the raw materials used by these facilities. Thus, raw material characteristics and product grades are some of the factors affecting pollution load (Oketola and Osibanjo, 2009b).

\subsection{Pollution load assessment by effluent analysis}

The results of the composite untreated effluent samples collected from the production line of the facilities are presented in Tables 4 and 5, respectively. The result of effluents analysis showed varying concentration of some of the parameters such as heavy metals, COD etc., which are above the permissible limits of Federal Ministry of Environment, (FEPA, 1998) for effluent discharge thus indicating gross pollution. The values of some of the parameters obtained could be attributed to the production processes, raw material characteristics etc.

\begin{tabular}{|c|c|c|c|c|c|c|c|c|}
\hline $\begin{array}{c}\text { Industrial Code } \\
\text { /Parameters }\end{array}$ & BGR & CAP & UST $^{1}$ & TTP $^{1}$ & WSY & LOP & IGM & AET \\
\hline $\begin{array}{c}\text { Sampling time } \\
\text { (n) }\end{array}$ & $\mathbf{4}$ & $\mathbf{5}$ & $\mathbf{2}$ & $\mathbf{2}$ & $\mathbf{3}$ & $\mathbf{2}$ & $\mathbf{2}$ & $\mathbf{5}$ \\
\hline Parameters & & & & & & & & \\
\hline Temp0C & $30.3 \pm 1.7$ & $29.2 \pm 1.8$ & 45 & $33 \pm 1.4$ & $46.3 \pm 7.8$ & $36 \pm 1.4$ & $29.5 \pm 0.7$ & $30.5 \pm 0.7$ \\
\hline pH & $7.62 \pm 0.5$ & $6.32 \pm 0.5$ & $6.75 \pm 0.1$ & $5.75 \pm 0.1$ & $9.6 \pm 1.0$ & $6.85 \pm 0.6$ & $11.3 \pm 0.0$ & $10.8 \pm 0.9$ \\
\hline $\begin{array}{c}\text { Turbidity } \\
(\mathrm{NTU})\end{array}$ & $4.15 \pm 0.3$ & $3.53 \pm 0.5$ & $\mathrm{ND}$ & $\mathrm{ND}$ & $\mathbf{0 . 3 1} \pm 0.04$ & $1,230 \pm 360$ & $\mathrm{ND}$ & $0.72 \pm 0.1$ \\
\hline $\begin{array}{c}\text { Conductivity } \\
(\mu \mathrm{s} / \mathrm{cm})\end{array}$ & $2210 \pm 410$ & $810 \pm 85$ & $104 \pm 5.7$ & $260 \pm 14$ & $0.31 \pm 0.04$ & $305 \pm 78$ & $2,700 \pm 28$ & $3550 \pm 780$ \\
\hline TSS (mg/L) & $9.65 \pm 2.8$ & $1.40 \pm 0.8$ & $0.28 \pm 0.3$ & $0.05 \pm 0.01$ & $0.14 \pm 0.1$ & $301 \pm 66$ & $1.55 \pm 1.3$ & $2.33 \pm 1.4$ \\
\hline
\end{tabular}




\begin{tabular}{|c|c|c|c|c|c|c|c|c|}
\hline $\begin{array}{l}\text { Oil \& Grease } \\
(\mathrm{mg} / \mathrm{L})\end{array}$ & $3.42 \pm 8.8$ & $6.30 \pm 1.5$ & $104 \pm 5.7$ & $260 \pm 14$ & $2,400 \pm 400$ & $91.2 \pm 30$ & $0.34 \pm 0.4$ & $34.3 \pm 30$ \\
\hline $\begin{array}{l}\text { Total Alkalinity } \\
(\mathrm{pH} 4.3)(\mathrm{mg} / \mathrm{L})\end{array}$ & $863 \pm 570$ & $650 \pm 270$ & $0.37 \pm 0.4$ & ND & $1.0 \pm 0.4$ & $32.6 \pm 46$ & $505 \pm 710$ & $3,730 \pm 2,400$ \\
\hline $\begin{array}{c}\text { Total Acidity } \\
(\mathrm{pH} 8.3)(\mathrm{mg} / \mathrm{L})\end{array}$ & $813 \pm 97$ & $602 \pm 120$ & $41.1 \pm 6.7$ & $67.9 \pm 10$ & $7931.0 \pm 61$ & $40.5 \pm 31$ & ND & $2,070 \pm 1,300$ \\
\hline $\begin{array}{c}\text { Methyl Orange } \\
\text { Acidity (pH 3.7) } \\
\text { (mg/L) }\end{array}$ & $293 \pm 590$ & ND & $34.9 \pm 32$ & ND & $147 \pm 120$ & ND & ND & - \\
\hline $\begin{array}{l}\text { Total Hardness } \\
\quad(\mathrm{mg} / \mathrm{L})\end{array}$ & $78.7 \pm 28$ & $58.8 \pm 20$ & $222.6 \pm 300$ & $6.27 \pm 1.0$ & $376 \pm 530$ & $80.5 \pm 63$ & $35.9 \pm 43$ & $246 \pm 350$ \\
\hline $\mathrm{Cl}^{-}(\mathrm{mg} / \mathrm{L})$ & $82.2 \pm 38$ & $33.6 \pm 10$ & $8.57 \pm 4.1$ & $1.79 \pm 0.1$ & $36.7 \pm 18$ & $9.06 \pm 0.5$ & $2.44 \pm 1.0$ & $21.1 \pm 38$ \\
\hline $\mathrm{SO}_{4}{ }^{2-}(\mathrm{mg} / \mathrm{L})$ & $106 \pm 53$ & $855 \pm 780$ & $46.1 \pm 2.7$ & $1.19 \pm 0.1$ & $1,180 \pm 680$ & $37.4 \pm 49$ & $199 \pm 120$ & $717 \pm 520$ \\
\hline $\mathrm{PO}_{4}^{3}-(\mathrm{mg} / \mathrm{L})$ & $94.5 \pm 20$ & $46.2 \pm 17$ & ND & ND & $\begin{array}{ll}7 & 7.0 \pm 6.1\end{array}$ & $10.5 \pm 9.6$ & $12.0 \pm 17$ & $47.5 \pm 14$ \\
\hline $\mathrm{NO}_{3^{-}}(\mathrm{mg} / \mathrm{L})$ & $2.12 \pm 1.4$ & ND & ND & ND & $0.8 \pm 0.7$ & $0.11 \pm 0.1$ & ND & ND \\
\hline $\mathrm{DO}(\mathrm{mg} / \mathrm{L})$ & ND & ND & $7.50 \pm 1.4$ & $6.80 \pm 0.1$ & ND & ND & ND & $80 \pm 1.8$ \\
\hline $\mathrm{COD}(\mathrm{mg} / \mathrm{L})$ & $1700 \pm 630$ & $642 \pm 390$ & $130 \pm 6.4$ & $621 \pm 43$ & $783 \pm 86$ & $22,160 \pm 95$ & $897 \pm 7.1$ & $159 \pm 130$ \\
\hline $\mathrm{BOD}_{5}(\mathrm{mg} / \mathrm{L})$ * & $23.4 \pm 2.9$ & $20.3 \pm 7.7$ & $10.5 \pm 3.0$ & $0.48 \pm 0.04$ & $4.56 \pm 0.4$ & $54.5 \pm 18$ & ND & $3.95 \pm 1.9$ \\
\hline $\mathrm{Ca}(\mathrm{mg} / \mathrm{L})$ & $15.3 \pm 5.9$ & $15.6 \pm 15$ & $0.34 \pm 0.2$ & $1.04 \pm 0.02$ & $14.6 \pm 15$ & $53.8 \pm 65$ & $38.2 \pm 19$ & $0.02 \pm 0.04$ \\
\hline $\mathrm{Mg}(\mathrm{mg} / \mathrm{L})$ & $9.85 \pm 9.2$ & $5.77 \pm 7.1$ & $53.8 \pm 73$ & $0.78 \pm 0.1$ & $82.3 \pm 140$ & $14.6 \pm 15$ & $0.73 \pm 1.0$ & $60.0 \pm 85$ \\
\hline $\mathrm{Pb}(\mathrm{mg} / \mathrm{L})$ & $2.01 \pm 4.0$ & $12.4 \pm 15$ & $3.07 \pm 4.3$ & ND & $9.07 \pm 16$ & $0.22 \pm 0.3$ & ND & $19.0 \pm 23$ \\
\hline $\mathrm{Ni}(\mathrm{mg} / \mathrm{L})$ & $0.73 \pm 0.5$ & $0.52 \pm 0.8$ & $0.10 \pm 0.1$ & $0.35 \pm 0.1$ & ND & $0.1 \pm 0.1$ & $0.6 \pm 0.8$ & $0.48 \pm 0.8$ \\
\hline $\mathrm{Cd}(\mathrm{mg} / \mathrm{L})$ & $0.78 \pm 1.1$ & $1.77 \pm 1.3$ & $0.11 \pm 0.2$ & ND & $0.09 \pm 0.2$ & ND & ND & $0.44 \pm 0.6$ \\
\hline $\mathrm{Cr}(\mathrm{mg} / \mathrm{L})$ & $0.53 \pm 0.4$ & $0.41 \pm 0.3$ & $0.18 \pm 0.2$ & $0.05 \pm 0.01$ & $0.18 \pm 0.1$ & ND & $0.2 \pm 0.3$ & $0.19 \pm 0.3$ \\
\hline $\mathrm{Fe}(\mathrm{mg} / \mathrm{L})$ & $8.80 \pm 6.4$ & $4.56 \pm 6.4$ & $7.3 \pm 10$ & ND & $8.27 \pm 7.2$ & $1.40 \pm 2.0$ & $4.9 \pm 6.9$ & $8.96 \pm 12$ \\
\hline $\operatorname{Mn}(\mathrm{mg} / \mathrm{L})$ & $2.71 \pm 2.2$ & $1.02 \pm 0.9$ & ND & $0.23 \pm 0.3$ & ND & $0.06 \pm 0.1$ & $0.27 \pm 0.4$ & $0.98 \pm 1.5$ \\
\hline $\mathrm{Zn}(\mathrm{mg} / \mathrm{L})$ & $0.15 \pm 0.1$ & $0.02 \pm 0.04$ & $1.00 \pm 1.4$ & ND & $0.01 \pm 0.02$ & $0.01 \pm 0.01$ & ND & $0.06 \pm 0.1$ \\
\hline $\mathrm{Cu}(\mathrm{mg} / \mathrm{L})$ & $20.7 \pm 14$ & $8.48 \pm 7.0$ & $2.70 \pm 2.2$ & $0.30 \pm 0.1$ & $2.54 \pm 0.6$ & $7.8 \pm 7.8$ & $4.98 \pm 7.0$ & $14.3 \pm 6.5$ \\
\hline Co $(\mathrm{mg} / \mathrm{L})$ & $0.29 \pm 0.1$ & $0.14 \pm 0.1$ & $0.04 \pm 0.1$ & $0.02 \pm 0.01$ & $0.23 \pm 0.2$ & ND & $0.14 \pm 0.2$ & $0.25 \pm 0.1$ \\
\hline TOTAL $(\mathrm{mg} / \mathrm{L})$ * & 36.7 & 20.8 & 14.5 & 0.95 & 20.4 & 9.59 & 11.1 & 44.6 \\
\hline
\end{tabular}

Note: * Parameters compared with IPPS pollution load ${ }^{1}$ cooling water

Table 4. Mean concentration and standard deviation of physico-chemical parameters of untreated effluent from the selected industries 
Assessment of Industrial Pollution Load in Lagos, Nigeria

\begin{tabular}{|c|c|c|c|c|c|c|c|}
\hline $\begin{array}{c}\text { Industrial } \\
\text { Code/Parameters }\end{array}$ & $\begin{array}{c}\text { BGR } \\
(n=2)\end{array}$ & $\begin{array}{c}\text { CAP } \\
(n=2)\end{array}$ & $\begin{array}{l}\text { WSY } \\
(n=2)\end{array}$ & $\begin{array}{c}\text { LOP } \\
(n=2)\end{array}$ & $\begin{array}{l}\text { IGM } \\
(n=3)\end{array}$ & $\begin{array}{c}\text { AET } \\
(n=2)\end{array}$ & $\begin{array}{l}\text { FMENV } \\
\text { LIMIT }\end{array}$ \\
\hline \multicolumn{8}{|l|}{ Parameters } \\
\hline $\mathrm{Temp}^{0} \mathrm{C}$ & $30 \pm 2.8$ & $28.8 \pm 3.2$ & $47.8 \pm 1.8$ & $30.8 \pm 0.4$ & $35 \pm 2$ & $29.5 \pm 0.7$ & \\
\hline $\mathrm{pH}$ & $7.3 \pm 0.3$ & $8.2 \pm 0.0$ & $9.85 \pm 0.2$ & $8.45 \pm 1.1$ & $9.03 \pm 0.3$ & $10.3 \pm 0.9$ & $6.5-9.0$ \\
\hline Turbidity (NTU) & $0.05 \pm 0.01$ & $0.06 \pm 0.01$ & $0.44 \pm 0.2$ & $137 \pm 52$ & ND & $0.41 \pm 0.03$ & \\
\hline Conductivity ( $\mu \mathrm{s} / \mathrm{cm}$ ) & $545 \pm 92$ & $2,300 \pm 140$ & $4,500 \pm 710$ & $289 \pm 150$ & $5,670 \pm 610$ & $\begin{array}{c}3,400 \pm 57 \\
0\end{array}$ & \\
\hline TSS (mg/L) * & $0.23 \pm 0.02$ & $0.32 \pm 0.1$ & $0.37 \pm 0.2$ & $32.0 \pm 9.9$ & $0.44 \pm 0.1$ & $1.91 \pm 1.3$ & \\
\hline Oil \& Grease (mg/L) & $0.30 \pm 0.03$ & $0.03 \pm 0.01$ & $19.2 \pm 3.8$ & $4.79 \pm 1.0$ & $9.19 \pm 6.8$ & $3.16 \pm 0.4$ & \\
\hline $\begin{array}{l}\text { Total Alkalinity (pH 4.3) } \\
\text { (mg/L) }\end{array}$ & $293 \pm 57$ & $572 \pm 97$ & $1,350 \pm 440$ & $131 \pm 56$ & $2,880 \pm 170$ & $\begin{array}{c}1,720 \pm 1,1 \\
00\end{array}$ & \\
\hline $\begin{array}{c}\text { Total Acidity ( } \mathrm{pH} 8.3) \\
(\mathrm{mg} / \mathrm{L})\end{array}$ & $136 \pm 130$ & $60 \pm 85$ & $220 \pm 75$ & $9.16 \pm 1.8$ & $76.1 \pm 16$ & ND & \\
\hline Total Hardness (mg/L) & $118 \pm 67$ & $44.5 \pm 20$ & $32.1 \pm 25$ & $22.0 \pm 8.5$ & $207 \pm 330$ & $1.57 \pm 2.2$ & \\
\hline $\mathrm{Cl}^{-}(\mathrm{mg} / \mathrm{L})$ & $31.9 \pm 0.2$ & $7.62 \pm 8.8$ & $46.4 \pm 66$ & $9.34 \pm 6.4$ & $127 \pm 31$ & $55.1 \pm 68$ & 600 \\
\hline $\mathrm{SO}_{4}^{2-}(\mathrm{mg} / \mathrm{L})$ & $103 \pm 16$ & $471 \pm 83$ & $303 \pm 84$ & $36.4 \pm 36$ & $111 \pm 32$ & $\begin{array}{c}1,100 \pm 89 \\
0\end{array}$ & \\
\hline $\mathrm{PO}_{4}^{3-}(\mathrm{mg} / \mathrm{L})$ & 8. $85 \pm 5.2$ & ND & $25.5 \pm 21$ & $3.14 \pm 1.0$ & $8.93 \pm 7.7$ & $43.5 \pm 30$ & \\
\hline $\mathrm{DO}(\mathrm{mg} / \mathrm{L})$ & $3.75 \pm 3.5$ & ND & ND & $0.75 \pm 1.5$ & $6.5 \pm 1.3$ & $1.75 \pm 2.5$ & \\
\hline $\mathrm{COD}(\mathrm{mg} / \mathrm{L})$ & $1450 \pm 92$ & $1,030 \pm 250$ & $1,140 \pm 510$ & $97.4 \pm 6.6$ & $363 \pm 260$ & $909 \pm 9.9$ & 80.0 \\
\hline $\mathrm{BOD}_{5}(\mathrm{mg} / \mathrm{L})$ * & $27.0 \pm 1.1$ & $16.1 \pm 2.7$ & $60.1 \pm 11$ & $21.8 \pm 8.5$ & $10.2 \pm 11$ & $6.55 \pm 1.0$ & 30.0 \\
\hline $\mathrm{Ca}(\mathrm{mg} / \mathrm{L})$ & $16.3 \pm 16$ & $2.34 \pm 0.1$ & $5.31 \pm 6.5$ & $10.9 \pm 13$ & $2.4 \pm 0.4$ & ND & \\
\hline $\operatorname{Mg}(\mathrm{mg} / \mathrm{L})$ & $18.7 \pm 6.6$ & $9.38 \pm 4.8$ & $4.55 \pm 2.1$ & $5.38 \pm 3.7$ & $48.8 \pm 79$ & $0.38 \pm 0.5$ & \\
\hline $\mathrm{Pb}(\mathrm{mg} / \mathrm{L})$ & $3.27 \pm 4.6$ & $4.7 \pm 6.7$ & $6.35 \pm 9.0$ & $7.0 \pm 9.9$ & $0.28 \pm 0.4$ & ND & $<1.0$ \\
\hline $\mathrm{Ni}(\mathrm{mg} / \mathrm{L})$ & $2.8 \pm 0.6$ & $1.20 \pm 0.3$ & $0.90 \pm 0.1$ & ND & $0.67 \pm 1.2$ & $0.8 \pm 1.1$ & $<1.0$ \\
\hline $\mathrm{Cd}(\mathrm{mg} / \mathrm{L})$ & $0.47 \pm 0.7$ & ND & $0.97 \pm 1.4$ & ND & $1.64 \pm 1.6$ & $0.15 \pm 0.1$ & $<1.0$ \\
\hline
\end{tabular}




\begin{tabular}{|c|c|c|c|c|c|c|c|}
\hline $\mathrm{Cr}(\mathrm{mg} / \mathrm{L})$ & $0.23 \pm 0.3$ & $0.14 \pm 0.1$ & $0.46 \pm 0.1$ & $0.23 \pm 0.3$ & $0.1 \pm 0.2$ & $0.29 \pm 0.4$ & $<1.0$ \\
\hline $\mathrm{Fe}(\mathrm{mg} / \mathrm{L})$ & $10.9 \pm 3.3$ & $0.6 \pm 0.9$ & $6.5 \pm 9.2$ & $4.18 \pm 5.7$ & $60.5 \pm 66$ & $61.1 \pm 61$ & 20.0 \\
\hline $\mathrm{Mn}(\mathrm{mg} / \mathrm{L})$ & $\mathrm{ND}$ & $\mathrm{ND}$ & $0.08 \pm 0.1$ & $0.06 \pm 0.1$ & $13 \pm 6.7$ & $\mathrm{ND}$ & 5.0 \\
\hline $\mathrm{Zn}(\mathrm{mg} / \mathrm{L})$ & $0.11 \pm 0.6$ & $0.20 \pm 0.3$ & $0.01 \pm 0.01$ & $0.12 \pm 0.2$ & $0.1 \pm 0.1$ & $0.07 \pm 0.1$ & $<1.0$ \\
\hline $\mathrm{Cu}(\mathrm{mg} / \mathrm{L})$ & $9.21 \pm 7.4$ & $8.03 \pm 4.6$ & $3.18 \pm 4.5$ & $1.81 \pm 2.4$ & $11.5 \pm 10$ & $6.85 \pm 2.0$ & $<1.0$ \\
\hline $\mathrm{Co}(\mathrm{mg} / \mathrm{L})$ & $0.32 \pm 0.4$ & $0.15 \pm 0.2$ & $0.16 \pm 0.1$ & $\mathrm{ND}$ & $0.11 \pm 0.2$ & $0.14 \pm 0.03$ & $<1.0$ \\
\hline TOTAL (mg/L) & $\mathbf{2 8 . 7}$ & $\mathbf{1 6 . 7}$ & $\mathbf{1 9 . 3}$ & $\mathbf{6 . 6 8}$ & $\mathbf{8 7 . 6}$ & $\mathbf{7 2 . 7}$ & \\
\hline
\end{tabular}

Note: * Parameters compared with IPPS pollution load

Table 5. Mean concentration and standard deviation of physico-chemical parameters of effluent discharged to the environment in the selected industries in Lagos

\subsection{Results of statistical analysis}

IPPS estimated pollution loads with respect to employment and total output in these industries were statistically analysed to ascertain the level of agreement between them. There is no significant difference between the pollution load estimated with respect to the two variables (i.e. employment and total output) at $\mathrm{p}>0.05$ in all the industries except in IGM, WSY, RLT, AWD, and AET. At the 0.05 level, the means are significantly different. IPPS pollution load was also compared with pollution load from conventional effluent analysis. There is no significant difference between them at $p>0.05$ in CAP, BGR, UST, TTP and AET while there is significant different between IPPS pollution load and pollution load from conventional effluent analysis in WSY. Hence, IPPS compared favourably with effluent analysis in most of the industries.

\section{Conclusion}

This study estimated pollution loads of some industries in Lagos using IPPS pollution intensities with respect to employment and total output. In most cases, the higher the total number of employees and total output, the higher the estimated pollution loads. There is no significant difference between the pollution loads estimated with respect to the two variables in all the industries except IGM where the two means are significantly different. IPPS pollution loads were also compared with pollution loads from conventional effluent analysis at $\mathrm{p}>0.05$. The two pollution loads compared favourably at this limit.

Application of IPPS in Lagos and most developing countries will no doubt enable the regulatory and monitoring agencies in such countries to focus on the most polluting industries. This will on the long run increase the level of enforcement since more time can be spent on the few polluting industries. This will also enable the policy makers in the developing countries to tackle industrial pollution since IPPS is a cheap means of assessing industrial pollution when compared to running scientific monitoring data gathering, analysis and assessment which is time consuming, expensive and resource intensive. 
Detailed information on employment and total output obtained from the fourteen industries studied revealed that in most cases, the higher the total number of employees and output, the higher the pollution loads by pollutant types except in TTP where the higher the total number of employees and total output, the lower the estimated pollution loads. This variation can be attributed to other factors which affect pollution loads. These are types and quantity of raw materials, process technology, product grade, efficacy of facility, and source type etc. Also, pollution load of the fourteen industries estimated with respect to employment and total output were compared statistically using t-test at $95 \%$ confidence interval and analysis of variance (ANOVA). At this level, the two means are not significantly different in CAP, BGR, TTP, FMI, UST, LOP, CLP, and APT while there was significant different in AWD, WSY, RLT, IGM, and AET. These can be attributed to the information and data supplied by these industries including process efficiency and efficacy of installed pollution control technology if any. For example, IGM with only 120 employees produced 1,170 ton/yr of total output while LOP with 200 employees have a total production capacity of 16.1 tons/yr which is significantly less than that of IGM.

The results of untreated effluent samples collected from these industries also revealed that most of the industries discharged untreated or partially treated effluent into the environment. Out of the 14 industries which data were available for this study, only $29 \%$ have effluent treatment plant which is operational, $36 \%$ have no effluent treatment plant while the remaining $36 \%$ operate dry process in which Effluent Treatment Plant (ETP) is not applicable. Unavailability of ETP in these industries could be attributed to the high cost of installing and maintaining an ETP, air pollution control devices, and weak enforcement of extant environmental regulations in Lagos.

Pollution load from conventional effluent analysis were compared with IPPS pollution load in these industries. There is no significant difference between them at $p>0.05$. IPPS pollution load of the selected industries compared favourably with pollution load from conventional effluent analysis in CAP, BGR, UST, TTP and AET. Enough data was not available from IGM and LOP. The exception was in WSY where there is significant difference between IPPS pollution load with respect to output and pollution load from conventional effluent analysis from effluent collected at the production line. Consequently, there was an agreement between effluent analysis or scientific monitoring and assessment and IPPS. Since IPPS compares favourably with scientific monitoring and analysis in these industries, IPPS therefore offers a cheap management tool for pollution load assessment in these industries; and directional basis for rapid policy intervention by government regulatory agencies in Lagos and other developing countries where pollution abatement technology is absent and level of enforcement is very low. It will enhance industrial pollution control in the developing countries where funding for environmental protection is lacking or grossly inadequate. The effectiveness of the intervening measures would significantly reduce the overall industrial pollution.

\section{References}

Akinsanya, C.K. (2003). Recent trends in the pollution load on the Lagos Lagoon. - Lagos state perspective. (A paper presented on ecological sustainable industrial development workshop organized by UNIDO).

Aguayo, F., Gallagher, P., and Gohzalez, A. (2001). Dirt is in the eye of the beholder: The World Bank air pollution intensities for Mexico. Global development and environment institute working paper, No. 01-07. 
APHA, 1992. Standard methods for the examination of water and wastewater. American Public Health Association, New York. 18th ed.

Arikawe-Akintola. J.O. (2002). The rise of industrialism in the Lagos area. In: Adefuye, A., Agiri, B., and Osuntokun, J. (Eds.).History of the peoples of Lagos state. Literamed publications limited, Lagos, Nigeria, pp. 102-116.

Arpad Horvath, Christ T. Hendrickson, Lester B. Lave, Francis C. McMichael, and Tse Sung Wu (1995). Toxic emissions indices for green design and inventory. Environ. Sci. Technol. 29, (2), 8 - 90A.

Bruce Kozak and Joseph Dzierzawski. (2003). Continuous casting of steel: basic principles. American iron and steel institute

Dasgupta, S., Lucas, E.B., and Wheeler, D., 2000. Small plants, pollution and poverty: new evidence from Brazil and Mexico. Policy research working paper, No. 2029.

Faisal, Islam, Rumi Shammiu, and Juhaina Junaid (1991). Industrial pollution in Bangladesh. Retrieved on July 24, 2003, from http:/ / www.worldbank.org/nipr

Federal Ministry of Environment, Housing and Urban Development (FMENV) (1998). Industrial pollution inventory study.

Hettige, H., Martin, P., Singh, M., and Wheeler, D. (1994). The Industrial Pollution Projection System (IPPS) policy research working paper, No. 1431, part 1 and 2.

Hettige, H., Martin, P., Singh, M., and Wheeler, D. (1995). The Industrial Pollution Projection System (IPPS) policy research working paper, No. 1431, Part 3.

Jeremy A.T. Jones (2003). Electric arc furnace steelmaking. American Iron and Steel Institute. Nupro Corporation

Manufacturer's Association of Nigeria (M.A.N.) (1991). Yearly economic review.

Miroslav Radojevic and Viadimir N. Bashkin. (1999). Practical environmental analysis. Royal Society of Chemistry.

Ogungbuyi, O.M. and Osho, Y.B. (2005). Study on Industrial Discharges to the Lagos Lagoon. Report Submitted by United Nations Industrial Development Organization (UNIDO), Country Service Framework Programme under the Ecological Sustainable Industrial Development Programme.

Onianwa, P. C. (1985). Accumulation, exchange and retention of trace heavy metal in mosses from southwest Nigeria. Ph. D. thesis, University of Ibadan, Ibadan, Nigeria.

Onyekwelu, I.U., Junaid, K.A., and Ogungbuyi, O.M. 2003. Recent trends in the pollution load on the Lagos Lagoon - A National perspective. Presented by Federal Ministryof Environment at the Ecological Sustainable Industrial Development Workshop. 2 - 20.

Oketola, A.A., and Osibanjo, O. (2009a). Estimating sectoral pollution load in Lagos by Industrial Pollution Projection System (IPPS): Employment versus Output. Toxicological \& Environmental Chemistry. 91, (5), 799-818.

Oketola, A.A., and Osibanjo, O. (2009b). Industrial pollution load assessment by Industrial Pollution Projection System (IPPS). Toxicological \& Environmental Chemistry. 91, (5), 989-997.

Taras J. Michael. (1950). Phenoldisulphonic acid method of determining nitrate in water. Anal Chem., 22, (8), 1020-102 


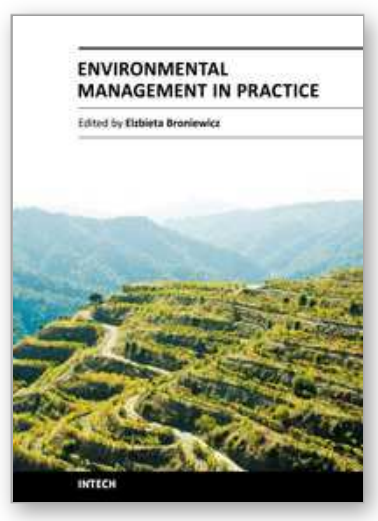

\section{Environmental Management in Practice}

Edited by Dr. Elzbieta Broniewicz

ISBN 978-953-307-358-3

Hard cover, 448 pages

Publisher InTech

Published online 21, June, 2011

Published in print edition June, 2011

In recent years the topic of environmental management has become very common. In sustainable development conditions, central and local governments much more often notice the need of acting in ways that diminish negative impact on environment. Environmental management may take place on many different levels - starting from global level, e.g. climate changes, through national and regional level (environmental policy) and ending on micro level. This publication shows many examples of environmental management. The diversity of presented aspects within environmental management and approaching the subject from the perspective of various countries contributes greatly to the development of environmental management field of research.

\section{How to reference}

In order to correctly reference this scholarly work, feel free to copy and paste the following:

Adebola Oketola and Oladele Osibanjo (2011). Assessment of Industrial Pollution Load in Lagos, Nigeria by Industrial Pollution Projection System (IPPS) versus Effluent Analysis, Environmental Management in Practice, Dr. Elzbieta Broniewicz (Ed.), ISBN: 978-953-307-358-3, InTech, Available from:

http://www.intechopen.com/books/environmental-management-in-practice/assessment-of-industrial-pollutionload-in-lagos-nigeria-by-industrial-pollution-projection-system-i

\section{INTECH}

open science | open minds

\author{
InTech Europe \\ University Campus STeP Ri \\ Slavka Krautzeka 83/A \\ 51000 Rijeka, Croatia \\ Phone: +385 (51) 770447 \\ Fax: +385 (51) 686166 \\ www.intechopen.com
}

\author{
InTech China \\ Unit 405, Office Block, Hotel Equatorial Shanghai \\ No.65, Yan An Road (West), Shanghai, 200040, China \\ 中国上海市延安西路65号上海国际贵都大饭店办公楼 405 单元 \\ Phone: +86-21-62489820 \\ Fax: +86-21-62489821
}


(C) 2011 The Author(s). Licensee IntechOpen. This chapter is distributed under the terms of the Creative Commons Attribution-NonCommercialShareAlike-3.0 License, which permits use, distribution and reproduction for non-commercial purposes, provided the original is properly cited and derivative works building on this content are distributed under the same license. 